\title{
A Brief Survey of Computational Models of Normal and Epileptic EEG Signals: A Guideline to Model-based Seizure Prediction
}

\author{
Farzaneh Shayegh $^{1,2}$, Rasoul AmirFattahi ${ }^{1,2}$, Saeid Sadri ${ }^{1,2}$, Karim Ansari-Asl $^{3}$ \\ ${ }^{1}$ Medical Image and Signal Processing Research Center, Isfahan University of Medical Sciences, Isfahan, Iran, ${ }^{2}$ Departments of Electrical and \\ Computer Engineering, Digital Signal Processing Laboratory, Isfahan University of Technology, Isfahan, ${ }^{3}$ Department of Electrical, Engineering \\ Faculty, Shahid Chamran University of Ahvaz, Ahvaz, Iran
}

\section{A B S T R A C T}

In recent decades, seizure prediction has caused a lot of research in both signal processing and the neuroscience field. The researches have tried to enhance the conventional seizure prediction algorithms such that the rate of the false alarms be appropriately small, so that seizures can be predicted according to clinical standards. To date, none of the proposed algorithms have been sufficiently adequate. In this article we show that in considering the mechanism of the generation of seizures, the prediction results may be improved. For this purpose, an algorithm based on the identification of the parameters of a physiological model of seizures is introduced. Some models of electroencephalographic (EEG) signals that can also be potentially considered as models of seizure and some developed seizure models are reviewed. As an example the model of depth-EEG signals, proposed by Wendling, is studied and is shown to be a suitable model.

Key words: Computational model, electroencephalographic signals, epilepsy, seizure prediction

\section{INTRODUCTION}

Epilepsy is second incident disease of the brain after a stroke that involves about $0.6-0.8 \%$ of the people. ${ }^{[1]}$ A sudden occurrence of the epileptic seizures makes them unbearable and thus limits the patients' life. One would think that patients with seizure do not have a normal life because of their sympathy for the unpredictable occurrence of seizures. Furthermore, although it is not proved, seizures may lead to brain injury. Fortunately, there are special drugs for specific patients, depending on the clinical and paraclinical symptoms. These drugs are advised based on the results of the researches carried out by neurophysiologists, epileptologists, and others. These researches are mainly conducted at a microscopic level, by experiments on animals. These are controlled experiments, and their results are so limited that the proposed drugs cannot cover all the possible conditions of seizure genesis. Thus, it is not astonishing if some patients' epilepsies are drug-resistant. Actually, for seizures with only a little difference from what is seen during in-vivo experiments, the drugs may not have their remedy effects. In fact about $25 \%$ of the epileptic patients suffer from intractable seizures. On the other hand, we should not neglect the side effects of the drugs.
For the patients, the final medical remedy is surgery, through which the areas of the brain involving seizure generation are removed. This operation may also be unsuccessful. In the opinion of engineers that deal with the brain as a system, if the main signals of a pre-seizure were recognized, then the on-time use of drugs, either oral or via injection or even some electrical stimulation, would improve both the above-mentioned problems. In the former, by ordering a sufficient dose at just the right time, the percent of the cured seizure will increase, and in the latter, this will notably decrease the amount of used drugs and mitigate the side effects. Similar to experiments that are conducted for discovering useful drugs, finding the critical times of using the drugs or electrical stimulation requires a good realization of the seizure mechanisms.

The field that focuses on the techniques of seeking the critical times of seizure procedure is called seizure prediction. In other words, a critical time or point at which a seizure can be turned around is the point that is likely to reach the seizure in the near future, but also soon enough to prevent its occurrence. Evidently, seizure prediction can be done based on the data of different levels from cell recordings to scalp EEG recordings. In order to be more clinically applicable, usually those seizure prediction studies are being used, 
wherein the scalp- or depth-EEG signals during pre-surgical evaluations are at the center of attention. Prediction of seizures based on cellular recordings may be more accurate, but nearly impossible for clinical use. If seizure prediction and the proper design of electrical stimulation procedures are successfully finalized, perhaps in the future, a special form of pacemaker will also be installed in the brain.

Since 1970 , seizure predictability based on scalp-EEG and depth-EEG signals has been studied. To date, results with very different promising and despairing reports are presented, sometimes based on extraction of similar features, but using different data. One would say that different types of seizures (generalized vs. focal and also from different locations) are predictable at different levels of accuracy. Furthermore, it seems that each individual may experience his/her seizures by a natural amount of predictability, like the fact that only some patients experience aura. Even different occurrences of seizures of a special person may require different predictions. These variable situations justify the various results obtained by researchers.

Indeed, when there are different mechanisms underlying each type of seizure and/or seizures of different patients, in spite of their similar manifestation, different techniques should be used for each seizure type and patient. These techniques are preferred to be designed based on their seizure genesis procedure. For example, it is concluded that it is not enough to consider the nonlinearity of the brain system and its features. For every seizure, the nonlinearity may have its own detail, and the knowledge about it may improve the results.

In this article, after a brief review of different seizure prediction algorithms and emphasizing on the contradictory results, the importance of state-of-the-art mechanism-based algorithms has been justified, and the essential requirement of such predication algorithms has been declared and abstractly described. It is worth mentioning that when we predict seizures based on a physiological model, with some parameters indicating cellular or neural network activities, it seems that we are predicting seizures from a closer point to neurons, but via signals that are easily recorded clinically.

\section{HISTORICAL REVIEW}

The seizure prediction concept was propounded as a preictal state detection at its birth. The seemingly separable seizure signals from the normal ones had made the on-hand matter very reachable and easy to perform. Thus, only some simple features based on linear assumption were used, ${ }^{[2-4]}$ and as the importance of the specificity of predictions had not been noticed in those days, the reliability of the results was overestimated. Later on, the features obtained according to linear assumptions were criticized, and to improve the sensitivity, they were replaced by nonlinear assumptions. ${ }^{[5-22]}$ Accordingly, some features, which largest Lyapunov exponents and correlation dimension are their pioneers, were had been proposed. However, later the researchers realized that in order to be applicable, seizure prediction algorithms should also be sufficiently specific. This new expectation showed that even by considering nonlinearity, the validity of the results could not be enhanced. The complexity of the nonlinear features was in favor of using linear ones. Therefore, nonlinearity of the performance of the brain was suspected. Subsequently, some special studies showed that the procedure was not linear ${ }^{[23]}$ and that weaknesses of the algorithms considering the nonlinear features had other reasons.

In the next steps, the bivariate features, based on using more information of the multi-channel signals, were proposed, and their better efficiency compared to univariate features was proved. $^{[24-35]}$ Synchronization ${ }^{[36-40]}$ was a well-known example of these bivariate features. Some algorithms based on choosing the optimal couple of channels were also proposed.

In 2009, a workshop on seizure prediction was held, in which the most important debates about seizure prediction were discussed. ${ }^{[41]}$ Evidently, it was declared that there was essential ambiguity in some features considered for the brain. For example, usually increment of the synchronization between two EEG channels was considered as a seizure predictor, but some neurologists rejected this result, mainly based on their investigations.

On the other hand, a careless look at the seizure phenomenon oversimplified the problem and this prevented researchers from proposing advanced prediction algorithms for the case.

Two recent viable fields of seizure prediction are as follows: ${ }^{[2]}$

1. The first involves specialists of pattern recognition. They believe that there is no single lone feature that can characterize pre-seizure signals, but somehow a number of features must be intermingled and composed, perhaps in opposite directions, such that all of their information can be gathered altogether. Furthermore, the preprocessing of the signals before feature extraction must be extensively considered; signal decomposition and artifact removal techniques are two examples. More powerful classifiers are also under investigation.

2. The second field mainly concerns the neuroscientists. They propose to make use of the dynamic mechanism underlying seizure generation, in order to predict seizures.

The second field is also advantageous, as, for discrimination between the types of epilepsies their mechanisms must be known. Thus, in this article we focus on the second field. 


\section{MODEL-BASED SEIZURE PREDICTION}

The goal of this article lies in describing the role of computational models in seizure prediction methods. The obstacles and the advantages will be introduced. The steps undertaken by a physiological model-based algorithm are listed as below:

1. Select the type of EEG signals, whether a scalp EEG, ECoG or depth-EEG and the local field potentials

2. Decide whether a special kind of seizure should be considered or all types of seizure are purposed

3. Study both the mechanism of seizure generation for the determined kind of seizure and the semiology of the relevant signals, and how to model them. Developed physiological model(s) already at hand can also be alternatively selected

4. Choose a suitable approach for identifying the hidden value of the physiological parameters of the model according to the recorded signals. This approach must indicate both the cost function and the optimization algorithm

5. Decide about the parameter sequences, whether they are predictors of seizures or not, and how to use them.

The main sections of this article deal with some important choices of steps 1-3: Some developed models of different EEG types are abstractly described. Subsequently, for a special model of a depth EEG signal, the system identification algorithms and some approaches for solving the optimization problem are illustrated.

\section{Type of Electroencephalogram Signals}

Electrical activity of the brain that is caused by firing of the neurons can be recorded in different ways. Neurons and the scalp are the two extreme points that electrical signals can be recorded from. However, intercellular and extracellular recordings are performed in the laboratory. Depth-EEG signals are captured by needle electrodes that are implanted in the cortex. Usually neurologists use these signals to localize, more precisely, the area of seizure activity, as a complementary diagnosis technique, just before surgery. ECoG signals are recorded from the surface of the cortices, using some grid or strip electrodes, and EEG signals are recorded from the scalp.

All of these signals somehow represent the local field potential of the brain areas where the electrodes are located. The farther we go from the source of the signals (brain), the electrodes integrate voltages of a larger population of neurons. Furthermore, the propagation path affects the electrical waves and the unavoidable interferences make the signals more noisy and susceptible to artifacts. Thus, there is a tradeoff between predictions of seizures based on signals of different levels. For example, a prediction based on a depth-EEG signal is easier to perform than that on surface EEG signals, but evidently its recording is an invasive act.

\section{Which Kind of Seizure is to be Noted?}

The predictability of different types of the seizure is not equally strange to neurologists and epileptologists. For some types of the seizures there is no definite fact about the phenomenon that leads to the seizure. Generalized seizures and absence seizures are among this category. The role of the non-cortical regions is necessary, but unknown, in these types of seizures. On the contrary, there is nearly a consensus about the mechanism of occurrence of some seizures, like focal epilepsies and specially Mesial Temporal Lobe Epilepsy (MTLE). It is perhaps due to the fact that a large percent of research about seizure mechanism is entitled to MTLE. Thus, in order to predict a special kind of seizure, its own related model must be used. If a prediction method independent of the type of seizure is required, either a more general method or a heuristic approach may be used.

\section{Review of the Models of Electroencephalogram Signals}

Now we try to give a quick review of some models that can be useful in this context. Before that, let us illustrate the perquisites of a model to be used as a candidate for modelbased seizure prediction algorithms.

To begin with, suppose thatwe have a model at hand that is capable of producing signals of different activities of the brain, according to the selected EEG signal. Note that such a model must inevitably be a stochastic model, to be able to imitate the natural randomness of the EEG signals. Moreover, suppose that what causes variation in the activity types is a set of parameters, and the model relates the properties of the signals to some physiological parameters. At its extreme (the ideal situation) assume that the model is managing an injective relation between the parameter vector and the model output. Now, each observed EEG signal can be assumed to be a model output of a special parameter value. Under such conditions, we can find the relevant parameter vector of each observed signal using a parameter identification process.

The sketch of the problem mentioned above can inspire the required situations for a model to be applied in seizure prediction:

1. We do not expect of the real models to produce any possible activity of EEG signals; but at least, some common activities must be produced by the model. As the model will be used for seizure prediction, in addition to normal EEG signals, seizure-related EEG activities, including high frequency signals, must be produced by the model. 
2. As the number of the model parameters, and accordingly, the dimension of the parameter vector increases, solving of the optimization problem will be more complicated and time consuming. This will be the reason for making us exclude the microscopic models that possess a number of parameters to control the type of activity of the model output; although lumped parameter models are preferable.

3. The expectation of the model to simulate a one-to-one relation between the parameter vector and the model output is too optimistic. Sometimes, especially when the model is stochastic, some acceptable amount of error must be tolerated.

Thus, every model must be evaluated based on the abovementioned points, to check whether it is a suitable candidate of seizure prediction or not.

In the next sections some intuitive notions about the elementary neural mechanisms and modeling of neuronal populations are described. It is useful to give a very simplified description of an isolated neuron model and what is called a neural population model.

\section{MICROSCOPIC (DETAILED) MODELS}

The milestone of brain activities is the neuron. There is a pile of neurons in both the central and peripheral nervous systems, whose most important specificities are their interactions, without which the function of the brain would be impossible. Thus, at first glance, modeling the behavior of individual neurons and also their connections is necessary. Accordingly, thousands of these neurons can be used to model the potentials of local fields by setting suitable relations among them.

Modeling individual neurons is the most important precedent in the field of expressing neurophysiological phenomena, by some mathematical and computational equations. Let us point out that synapses are the sites of interface between neurons. At the synapses neurons stimulate other neurons that are called efferent neurons. Synapses connect the soma of post-synaptic neurons to the dendrites of pre-synaptic ones. These dendrites behave like transmission lines that transmit electric voltage to on/ off pulses proportional to the amplitude of this voltage. Soma produces a low pass filtering effect on the pulse of the synapses parts. Usually synapses are junctions of many neurons. The low-pass filter then converts a number of on pulses at the pre-synaptic neurons, say the rate of firing, to the membrane voltage of the soma. Furthermore, when a neuron is excited, it takes at least a refractory time to the next excitability of the neuron. The Hodgkin-Huxley model $^{[42]}$ is one of the most famous models for the neuron. The noisy and leaky integrate-and-fire neurons and a Poisson spike-train cell model are the other examples. Specialized models for hippocampal neurons are also proposed. ${ }^{[43,44]}$ These models mainly differ in the nonlinear function of the dendrite, the linear impulse response of the soma filter, and the modeling refractory phenomenon. The common factor is the stochastic modeling of some of these systems. These systems have special values for each kind of neuron according to their properties.

In the neuronal tissue (gray matter of the brain) the neurons can often be divided into two main families, the principal neurons and interneurons. These neurons are then connected in a similar manner to real neural networks. For example, principal neurons have the peculiarity of having a privileged spatial orientation, roughly perpendicular to the cortical surface (when viewed in the neocortex). Interneurons do not have this peculiarity. This has great significance for the interpretation of the potential measured by a sensor placed within a neural population.

The neurons of a neural network can be connected to each other in different lattice forms. Any change in the ratio of the number of excitatory to the number of inhibitory neurons, as well as the structure of the connected neurons and the relevant coefficients can change the type of activity of the model output. In other words, there are too many effective parameters that prevent the model to be suitably intractable. Thus, generally, microscopic models do not have the second property of the above-mentioned conditions, to be applied in seizure prediction algorithms.

Netoff ${ }^{45]}$ proposed the brain as a small world network in which all the cells are only coupled to their nearest neighbors, but small numbers of connections are broken and rewired to make long-distance connections at random locations. As more long-distance connections are added, the network loses the property that most connections are local, and the network looks much more random. We find a range of normal and epileptiform behaviors in the small-world network regimen, where few connections are necessary to connect any pair of neurons, but local connections still predominate. Bursting and seizing behaviors appear as a number of long-distance connections change. However, connectivity, chemical distances, and clustering coefficients are some features of small world networks that can be noted as compressed parameters of the model. ${ }^{[46]}$

\section{MESOSCOPIC (REDUCED) MODELS}

Another type of model, called the reduced model is available. In this type of model, the complexity of single neurons is down, so a few of them can build EEG signals. The cortex is modeled as a matrix, such that each component of it contains an excitatory and inhibitory mass of neurons. ${ }^{[47]}$ These models are mainly modified versions of the microscopic models that can lead to a better comprehension of cortex dynamics. 


\section{MACROSCOPIC MODELS}

To generate a better insight into the functional significance of the emergent dynamics, some form of simplification is of critical importance. The neuronal networks of the cortex can be considered as a spatially continuous network in which the properties of a bulk of neurons at a local region are summarized in some state variables that their values are a function of both time and space. These variables can typically be the mean firing rates or the mean values of the cell (soma) membrane potential, just as there is an average neuron at that location. This kind of model is called the neural mass model. Evidently, a nonlinear function at the average dendrite and linear function at the average soma is expected. Furthermore, the input of the neural mass models is usually a white noise, accounting the influence of all the neurons that are far away.

\section{The Elementary Macroscopic Models}

At the initial stages, the neural mass models dealt with neurons having just excitatory interactions. ${ }^{[48]}$ Later, the inhibitory interaction was incorporated. Wilson and Cowan $^{[49,50]}$ considered the excitatory and inhibitory properties of a neuronal mass as two distinct neuronal populations that were connected to each other. The state variable was the mean firing rate at time t. By the assumption of the homogeneous property of the underlying neuronal mass, in both models, a similar behavior was assumed for every location of it. The equations underlying these models were thus differential equations. These equations could easily be extended into partial differential equations, to account for the spatial properties of the neuronal mass. On the other hand, Amari ${ }^{[51,52]}$ proposed a spatial-temporal model with the mean soma membrane potential as a macroscopic state variable.

Both these models are oversimplified such that they are unrealistic in some cases. For example, in both the models, the mean low pass filter is assumed to be of the first order, which induces an instantaneous effect at the neuronal soma, instead of the fact that these effects steadily increase to their maximum levels. Nevertheless, all the later neural mass models, proposed up to now, are extensions of these models.

Although these models are the simplest models presented for a mass of neurons, their differential equations possess nonlinear terms as a result of the thresholding effect at the dendrites. Thus, no analytical solutions exist for these equations and they must be qualitatively analyzed. By assessment of the steady states (fixed points) and limit cycles, further insight can be gained into the properties of the dynamics of the cortex performance. There is no claim by these models about producing EEG signals, but if the parameters are suitably selected, sustained limit cycle activity and damped oscillatory behavior, in response to brief stimulating inputs, can be seen.

Subsequently, these models were extended to produce EEG signals that had sufficient dynamical complexity and empirical merit.

\section{Freeman's Model}

Freeman, in a hierarchal manner, ${ }^{[53]}$ tried to explain the electrocortical dynamics of the olfactory bulb and prepyriform cortex. At the lowest level a $\mathrm{KO}$ model exists in which there are neurons that are common in the input and output, but with no interaction between them. Unlike the Wilson and Cowan and Amari's models, this set is characterized by a third order differential linear equation as the 'pulse-to-wave' function of the average dendrite. At level $\mathrm{KI}$, the interactions of these $\mathrm{KO}$ sets are either excitatory or inhibitory: $\mathrm{KI}_{\mathrm{e}}$ and $\mathrm{KI}_{\mathrm{i}}$. Dense functional interaction between two KI sets forms the KII set. The KII set is equivalent to the Wilson and Cowan model and is schematically displayed in Figure 1. It is defined by four nonlinearly coupled third order differential equations. The nonlinearity represents the 'wave-to-pulse' function of the average soma. The neocortex can be viewed as one or many interconnected KII sets. A range of physiologically relevant and complex behaviors, for example, the generation of the gamma band $(>30 \mathrm{~Hz})$ and the oscillatory activity, can be simulated by this model.

Still Freeman is extending his theories to models the cortical phenomena realistically.

\section{Lopez da Silva's Model}

As a very simple and also realistic model of the alpha rhythm of the EEG signals, Lopes da Silva noticed that the response of the average soma to the pre-synaptic spikes is at the very least of the second order: ${ }^{[55,56]}$ The average membrane potential rises to a peak and then decays. This little, but realistic modification of the Wilson and Cowon model caused the model to produce a strong resonance in the alpha band range $(8-13 \mathrm{~Hz})$. Lopez da Silva is developing

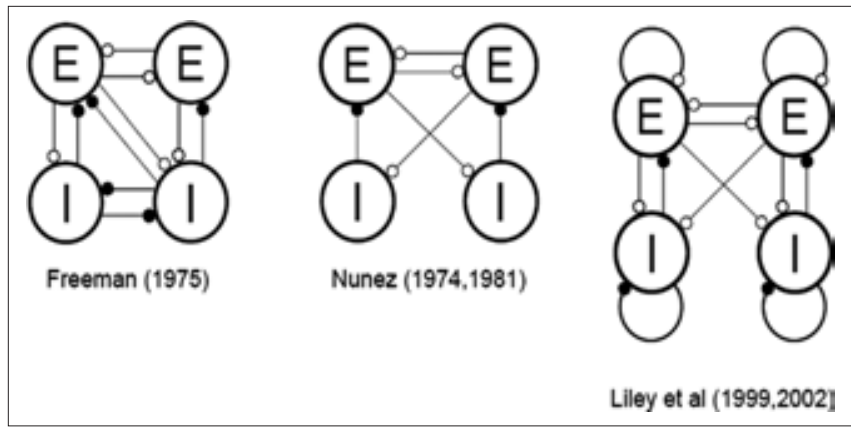

Figure I: Schematic outline of the connection topologies of a number of mean-field approaches to modeling the dynamics of EEG ${ }^{[54]}$ 
the models according to the different scenarios of seizure prediction. ${ }^{[57,58]}$

\section{Jansen's Model}

Jansen's model ${ }^{[59]}$ is based on Lopes da Silva's lumped parameter model. ${ }^{[55]}$ The basic idea behind these models is to make excitatory and inhibitory populations interact such that oscillations emerge. The wave-to-pulse operator at the average soma of a population of neurons and a linear pulse-to-wave conversion that are shown in Figure 2, are as follows:

$$
S(v)=\frac{2 e_{0}}{1+e^{r\left(v_{0}-v\right)}}
$$

and

$$
\begin{aligned}
& \dot{z}_{1}(t)=z_{2}(t) \\
& \dot{z}_{2}(t)=W x(t)-2 w z_{2}(t)-w^{2} z_{1}(t)
\end{aligned}
$$

where $x(t)$ and $z_{1}(t)$ are the input and output signals of this filter.

In each area of the cortex, there are two types of the neurons: Pyramidal cells and interneurons are mainly involved in the generation of paroxysmal activity in the isolated slab. ${ }^{[60]}$ Pyramidal cells are always excitatory, but both inhibitory and excitatory interneurons exist. Accordingly, three interacting subsets of neurons participate in this model. The main cells (i.e., pyramidal cells) receive a feedback from two other subsets, composed of local interneurons, either excitatory or inhibitory. ${ }^{[1]}$

\section{Nunez's Global Model}

In all cases, when the location is also considered as another variable in the differential equations, the local models of the neocortex will be global models. Also, the local models can be coupled to each other to model the global cortex. However, Nunez ${ }^{[62]}$ constructed a global model of electrorhythmogenesis by instantaneously considering short-term and long-term connections. A diagram of this model is given in Figure 1.

\section{Liley's Model}

Liley developed the basic theories of the macroscopic models by adding some new physiological facts. ${ }^{|63|}$ Thus, effects of the synaptic reversal potentials, which make the amplitude of the respective PSPs dependent on the ongoing postsynaptic/somatic membrane potential, and the transmission function of the axonal pulses in long range fibers, are added to the model. The structure of the Liley's model is presented in Figure 1. In other words, the effect of synaptic reversal potentials is changing the linear

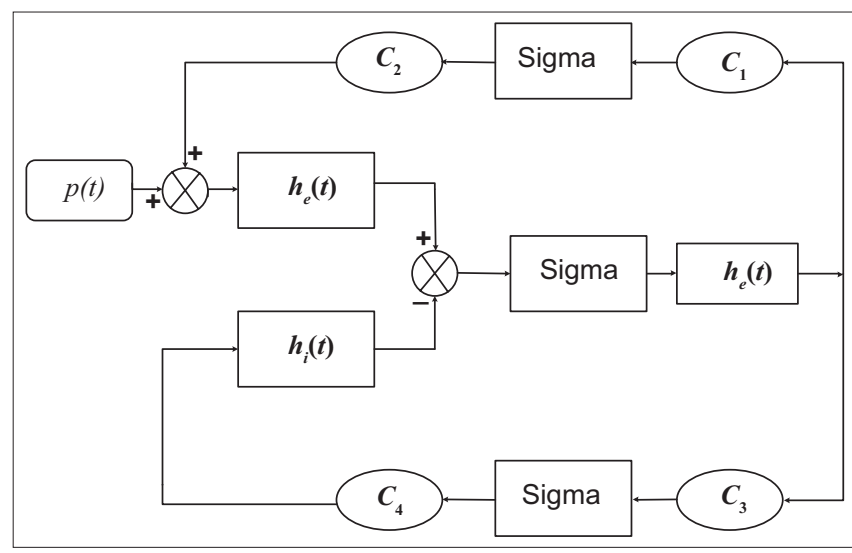

Figure 2: The Jansen neural mass model. Three different populations of neurons (excitatory pyramidal cells, excitatory principal cells, and inhibitory interneurons) compose a cortical area ${ }^{[6]]}$

relation between the mean soma membrane potential and the synaptic input, to an exponential one. The Liley model is capable of reproducing the main features of the spontaneous human EEG. In particular, the autonomous limit cycle and chaotic oscillatory activity in the alpha band $(8-13 \mathrm{~Hz})$ are easily produced.

\section{MODELS OF SEIZURE}

The above-mentioned models are proposed ones, to produce EEG-like signals. In all of them, interactions of excitatory and inhibitory population neurons play the main role. In this section, we continue with the models that can simulate seizure-like signals. From the seizing and bursting activity point of view, Liley and Wendling have specialized researches. Wendling has used the Lopes da Silva model to model an area of hippocampus, in order to model MTLE seizures. Liley has evaluated the role of the parameters of his model for the generation of unstable activities like seizures.

\section{Wendling's Model}

Based on the Jansen's model, Wendling tried to model the production of realistic epileptiform activity as a result of imbalance between excitatory and inhibitory synaptic gains (model parameters). Wendling observed that at some special values of parameters, normal EEG signals of the Jansen's model are replaced by spike activities or rhythmic signals, just like epileptic signals. ${ }^{[64]}$ Then, in order to represent fast EEG activity such as low-voltage, rapid discharges that are often observed in depth-EEG signals at seizure onset, Wendling developed a new version of the mentioned model, based on bibliographical material. ${ }^{[65]}$ A fourth subset was added to the model as an agent of a second class of inhibitory interneurons to raise the kinetics of the system. ${ }^{[65]}$ Actually, in addition to the usual $G A B A_{\mathrm{A}}$-mediated inhibition, $G A B A_{\mathrm{B}}$ neurotransmitters also had inhibitory effects. The kinetics of the $G A B A_{\mathrm{B}}$ were faster than those 
of $G A B A_{\mathrm{A}}$. The impulse responses of the excitatory, slow inhibitory, and fast inhibitory activities, respectively, were given by

$$
h_{e}(t)=\frac{A}{a} t \cdot e^{-\frac{t}{a}}, h_{i}(t)=\frac{B}{b} t \cdot e^{-\frac{t}{b}} \text { and } h_{g}(t)=\frac{G}{g} t \cdot e^{-\frac{t}{g}}, t>0
$$

The structure of this model is shown in Figure 3. The influence from the neighboring areas of the cortex is represented by an excitatory input $p(t)$ (modeled by the Gaussian white noise in the absence of target stimulation) in the model. The model output corresponds to the postsynaptic activity of the first subset, and is interpreted as an EEG signal. We call this model the depth-EEG model.

Thus, it is a stochastic model and its output corresponds to the postsynaptic activity of the first subset, and is interpreted as an EEG signal. This process is expressed with the following differential equations:

$$
\begin{aligned}
& \dot{y}_{0}(\mathrm{t})=y_{5}(\mathrm{t}) \\
& \dot{y}_{5}(\mathrm{t})=\frac{A}{a} S\left[y_{1}(\mathrm{t})-y_{2}(\mathrm{t})-y_{3}(\mathrm{t})\right]-\frac{2}{a} y_{5}(\mathrm{t})-\left(\frac{1}{a}\right)^{2} y_{0}(\mathrm{t}) \\
& \dot{y}_{1}(\mathrm{t})=y_{6}(\mathrm{t}) \\
& \dot{y}_{6}(\mathrm{t})=\frac{A}{a}\left\{p(\mathrm{t})+C_{2} S\left[C_{1} y_{0}(\mathrm{t})\right]\right\}-\frac{2}{a} y_{6}(\mathrm{t})-\left(\frac{1}{a}\right)^{2} y_{1}(\mathrm{t}) \\
& \dot{y}_{2}(\mathrm{t})=y_{7}(\mathrm{t}) \\
& \dot{y}_{7}(\mathrm{t})=\frac{B}{b} C_{4} S\left[C_{3} y_{0}(\mathrm{t})\right]-\frac{2}{\mathrm{~b}} y_{7}(\mathrm{t})-\left(\frac{1}{b}\right)^{2} y_{2}(t) \\
& \dot{y}_{3}(\mathrm{t})=y_{8}(\mathrm{t}) \\
& \dot{y}_{8}=\frac{G}{g} C_{7} S\left[C_{3} y_{0}(\mathrm{t})-C_{6} y_{4}(\mathrm{t})\right]-\frac{2}{\mathrm{~g}} y_{3}(\mathrm{t})-\left(\frac{1}{g}\right)^{2} y_{3}(\mathrm{t}) \\
& \dot{y}_{4}(\mathrm{t})=y_{9}(\mathrm{t}) \\
& \dot{y}_{9}=\frac{B}{b} S\left[C_{3} y_{0}(\mathrm{t})\right]-\frac{2}{b} y_{4}(\mathrm{t})-\left(\frac{1}{b}\right)^{2} y_{4}(\mathrm{t}) \\
& y_{\text {out }}(\mathrm{t})=y_{1}(\mathrm{t})-y_{2}(\mathrm{t})-y_{3}(\mathrm{t})
\end{aligned}
$$

In, ${ }^{[65]}$ the standard values of the linear system $\left(C_{1}\right.$ to $\left.C_{2}, a, b, \mathrm{~g}\right)$ and nonlinear function parameters $\left(\mathrm{e}_{0}, \mathrm{v}_{0}\right.$ and $r$ of (1)) are reported. These parameters are obtained through accurate experiments.

By changing the excitation and inhibition synaptic gain parameters ( $A, B$, and $G$, respectively) of the depthEEG model, six different types of EEG signals can be produced: ${ }^{[65]}$ Normal background, sustained discharge of spikes, low voltage rapid activity, slow quasi-sinusoidal activity, sporadic spikes, and slow rhythmic activity. Each of these type of signals can be met in the real world (normal and pathological) during different activities of the brain. The normal background EEG is observed during the normal activity of brain, either in healthy or epileptic

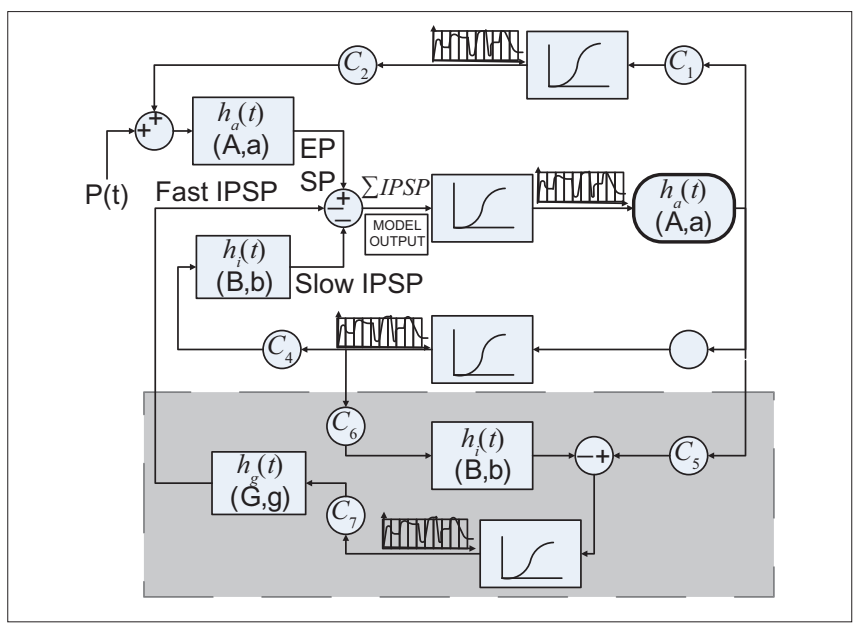

Figure 3: Organization of the depth-EEG model. Four neuronal subsets: Pyramidal cells, excitatory interneurons $(A)$, dendritic projecting interneurons with slow synaptic kinetics $\left(G A B A_{A \text {, slow }}: B\right)$, and somaticprojecting interneurons (the gray rectangle denotes these group of interneurons with its inward and outward connections) with faster synaptic kinetics $\left(G A B A_{A \text {, fast }}: G\right)$. The average pulse density of the afferent action potentials is changed into an average inhibitory or excitatory postsynaptic membrane potential, using a linear dynamic transfer function of impulse response $h_{i}(t), h_{e}(t), h_{g}(t)$, while this potential (physiologically in the post somatic part of the neurons) is converted into an average pulse density of firing of a post-synaptic neuron using a static nonlinear function ${ }^{[65]}$

people, called the interictal state in epileptic patients. Sporadic spikes are likely to appear on normal EEG signals, but may also be seen during some mental activity, even in healthy subjects.

Sustained discharge of spikes may be exposed at the onset of seizure, during seizure, and also during some nonpathological activities like evoked eye saccade. ${ }^{[66]}$ On the other hand, low-voltage rapid activity is usually encountered at the beginning of MTLE seizures. Furthermore, slow rhythmic activity is a possible characteristic of seizures and quasi-sinusoidal activity is just the kind of activity that is observed during the ictal state.

Figure 4 shows exemplar output signals of the model according to the different parameters (i.e., different models of the model space). A 3-D symbolic activity map for limited range of $A, B$, and $G$ is also displayed. Differential equations (3) are some stochastic differential equations and should accordingly be solved by a stochastic numerical algorithm, like the stochastic Runge-Kutta algorithm; but, based on, ${ }^{[61]}$ we accept the simplicity of solving our equations by the second order Runge-Kutta algorithm. However, we have considered the stochastic nature of $p(t)$ by first producing it with a random generator and then using its samples in the equations (3).

The white Gaussian noise is used as the hippocampcal modulatory input. The mean and variance of the input must be chosen such that the background activity parameters are produced using standard values; ${ }^{\mid 64]}$ to this end we selected $\mu=50$ and $\sigma=50$. 


\section{Liley Models the Effects of General Anesthetic Agents}

In Liley's proposal, the strength and time course of the cortical inhibitory-inhibitory and inhibitory-excitatory connectivity are the effective parameters of the model. The time course of these connectivities has not been considered in Wendling's theory. He tried ${ }^{[67]}$ to justify the influences of known pharmacologies via alterations in the strength and time course of the fast GABA ergic neurotransmission, and accordingly improved what is known about the mechanisms of seizure initiation and cessation. In other words, he described the generation of high frequency activities induced by general anesthetic agents from their effects on the neurotransmitters.

\section{Population Models for Absence Epilepsy}

The model proposed, ${ }^{[58]}$ is called as the macroscopic model by the authors, and is fairly close to a population model. It can explain the absence of epilepsy in an animal model (rats) that is switching from normal activity to the state of crisis and then return to normal. This is associated, in terms of the recorded field potentials, with a transition between spindle oscillations and spike-wave trains. The model is represented graphically in Figure 5a.

Unlike the model of hippocampus, this model, although macroscopic, has a thalamocortical loop, thus including populations of neurons in the following structures:

- A sub-cortical pyramidal cell population (PY)

- A subpopulation of inhibitory cortical cells $(I N)$

- A subpopulation of thalamocortical cells

- A subpopulation of cell thalamoreticulaires

The first two are located in the cortex and the other two in the thalamic region. Figure $5 \mathrm{~b}$ clearly shows the couplings:

- From $Y$ to $I N, T C$ and $R E$ (excitatory $A M P A$ )

- From IN to $P Y\left(G A B A\right.$ inhibitor and $\left.G A B A_{B}\right)$

- From TC to RE, IN and PY (excitatory AMPA)

- From $T C$ to $R E\left(G A B A_{A}\right.$ and excitatory $\left.G A B A_{B}\right)$

In addition there are three input signals:

- A cortical excitatory input on $P Y$

- An excitatory sensory input on $C T$

- An inhibitory input (GABA) on $E R$

Terms $A M P A, G A B A_{A}$, and $G A B A_{B}$ match the types of neurotransmitters.

Another difference in the model of a hippocampus is the presence of delays (delay lines) for links ascending from the thalamus to the cortex and the corresponding downlinks. In this model the $9 \mathrm{~Hz}$ discharge signals spontaneously emerge and soon disappear. This model

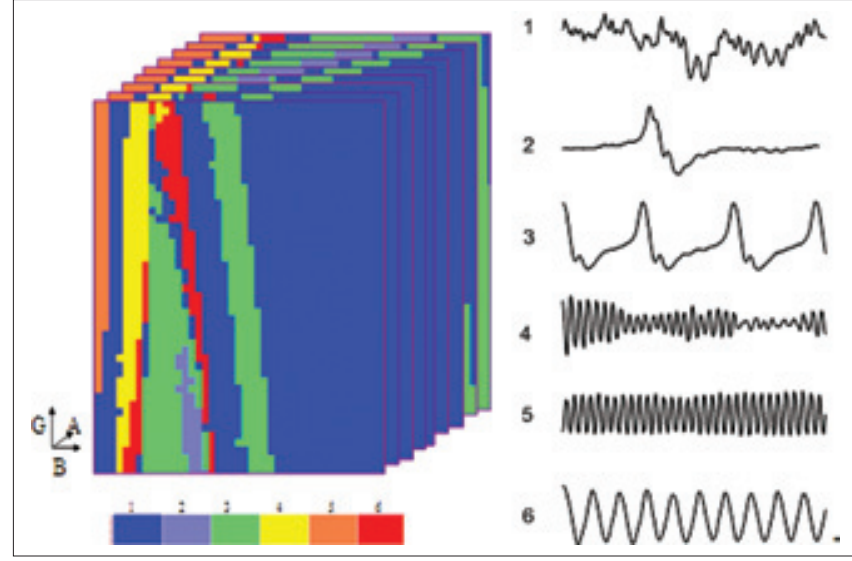

Figure 4: (a) Relation of the model output activity and the parameter vector value (Activity Map) of the depth-EEG model, and (b) six different kinds of the model output

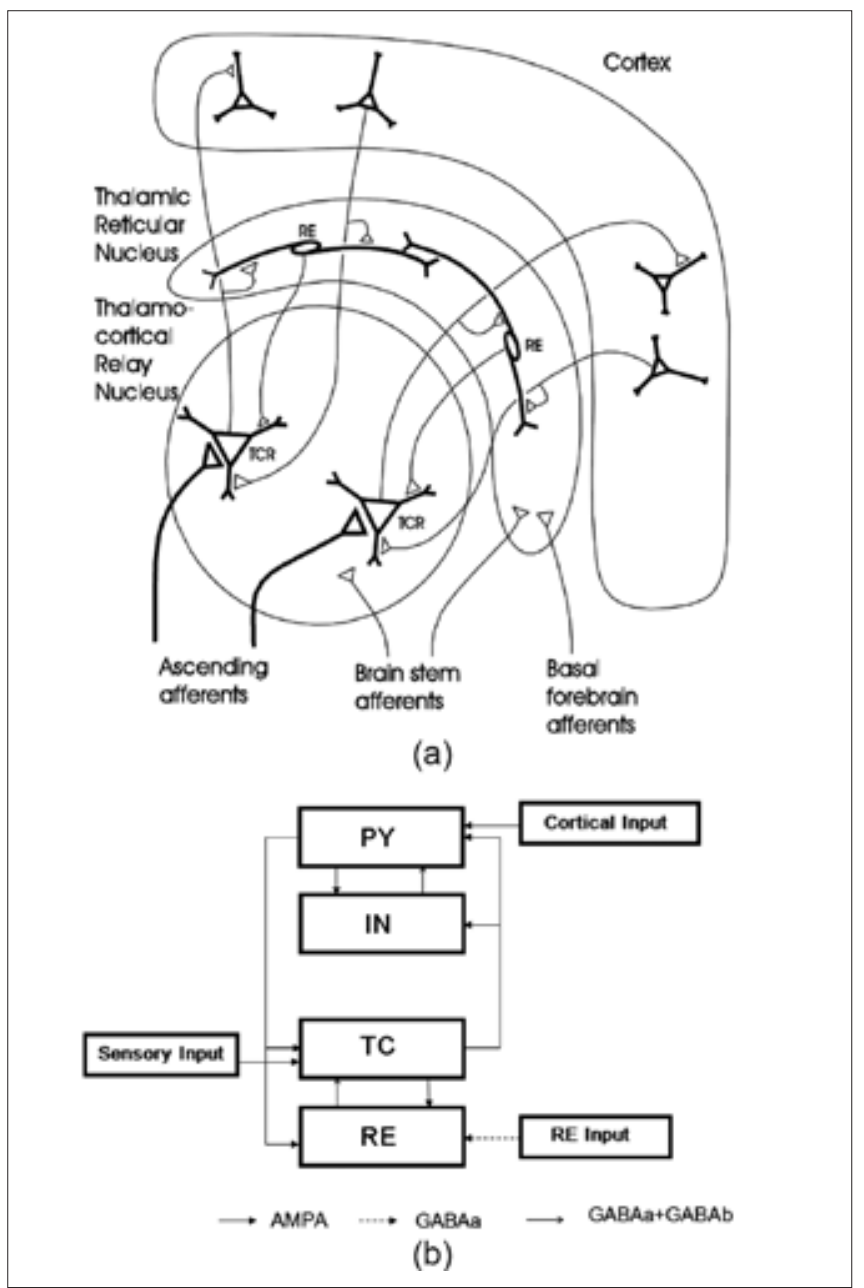

Figure 5: (a) The schematic of the absence model, cortex, thalamus, and the interface thalamocortical nucleus are displayed, (b) the couplings existing in the absence model ${ }^{[58]}$

corresponds to a strategy of population modeling, with special characteristics integrated in these populations, and it is a powerful tool for the investigation predictability of absence seizures. 


\section{IDENTIFICATION OF DEPTH- ELECTROENCEPHALOGRAM MODELS}

Now, each observed signal can be assumed as the output of, for example, the depth-EEG model, and the parameter vector that best reproduces it must be estimated. The parameter sequence corresponding to the recorded signal can improve the result of MTLE seizure prediction.

As equation (3) implies, the depth-EEG model is a stochastic model. Thus, in order to be identifiable, the observed signals should be of infinite samples. However, the maximum likelihood method ensures the acceptable estimation of parameters, based on sufficiently observing a large number of discrete samples. The loglikelihood, $p(y: \theta)$, is obtained using different approaches. ${ }^{\left.{ }^{668}\right]}$ For example the model output is analytically a Markov process, so that the likelihood can be approximated by using the optimal filter or Kalman Filter. Optimization of the obtained likelihood is conducted by using the simulated annealing technique.

On the other hand, as the calculations required for approximating the likelihood are too complicated and time consuming, an alternative method is used to compare the vector computed features identically from the observed signal and the model output. $\operatorname{In}^{[69]}$, the energies of different subbands are used as features. The genetic algorithm is applied to efficiently solve the optimization problem.

The obtained parameter sequences must then be assessed to indicate whether or not they can improve the specificity of the seizure prediction algorithms.

\section{CONCLUSION}

The mechanisms of the seizure must be well realized. As a direct route, identification of the parameters of a suitable physiological model is proposed. As availability of an appropriate model is the most important factor some of the most famous general models of EEG signals and also specialized seizure models are abstractly described. Among them, a model of the depth-EEG signals is more extensively assessed and the elementary steps of the modelbased prediction approach are mentioned. Furthermore, it is seen that the models are mainly based on local field potentials, so as to be useful in seizure prediction, based on surface EEG signals (to be clinically accessible). Some complementary modifications are needed, for example, local field potentials must be propagated through three shells containing the scalp, the skull, and the brain tissue, to form EEG signals. If this is modeled correctly, the EEG signal at each point of the surface will be influenced by the local field potentials of many points of the brain, which is a subject under investigation. ${ }^{[70,71]}$

\section{REFERENCES}

1. J. F. Annegers. The epidemiology of epilepsy. In: Wyllie E, editor. The Treatment of Epilepsy: Principles and Practice. Baltimore: Williams and Wilkins, pp. 165-72, 1996.

2. F. Mormann, R. G. Andrzejak, C. E. Elger, and K. Lehnertz. Seizure prediction: The long and winding road. Brain 130, pp. 1-20, 2007.

3. S.S. Viglione, and G.O. Walsh. Epileptic seizure prediction. Electroencephalogr Clin Neurophysiol 39, pp. 435, 1975.

4. Z. Rogowski, I. Gath, and E. Bental. On the prediction of epileptic seizures. Biol Cybern 42, pp. 9-15, 1981.

5. L. D. Iasemidis, J. C. Sackellares, H. P. Zaveri, and W. J. Williams. Phase space topography and the Lyapunov exponent of electrocorticograms in partial seizures. Brain Topogr 2, pp. 187-201, 1990.

6. J. Martinerie, C. Adam, M. Le Van Quyen, M. Baulac, S. Clemenceau, and B. Renault. Epileptic seizures can be anticipated by non-linear analysis. Nat Med 4, pp. 1173-6, 1998.

7. M. Le Van Quyen, J. Martinerie, M. Baulac, and F. Varela. Anticipating epileptic seizure in real time by a nonlinear analysis of similarity between EEG recordings. Neuroreport 10, pp. 2149-55, 1999.

8. M. Le Van Quyen, C. Adam, J. Martinerie, M. Baulac, S. Clemenceau, and F. Varela. Spatio-temporal characterization of non-linear changes in intracranial activities prior to human temporal lobe seizures. Eur J Neurosci 12, pp. 2124-34, 2000.

9. K. Lehnertz, and C.E. Elger. Spatio-temporal dynamics of the primary epileptogenic area in temporal lobe epilepsy characterized by neuronal complexity loss. Electroencephalogr Clin Neurophysiol 95, pp. 108-17, 1995.

10. P. Grassberger, T. Schreiber, and C. Schaffrath. Non-linear time sequence analysis. Int J Bifurcation Chaos 1, pp. 521-47, 1991.

11. C. E. Elger, and K. Lehnertz. Ictogenesis and chaos. In: P. Wolf, editor. Epileptic Seizures and Syndromes. London:J. Libbey and Co., pp. 547-52, 1994.

12. C. E. Elger, and K. Lehnertz. Prediction of epileptic seizures in humans from nonlinear dynamics analysis of brain electricalactivity. Eur J Neurosci 10, pp. 786-9, 1998.

13. K. Lehnertz, and C. E. Elger. Can epileptic seizures be predicted? Evidence from nonlinear time series analyses of brain electrical activity. Phys Rev Lett 80, pp. 5019-23, 1998.

14. K. Lehnertz, G. Widman, and R. G. Andrzejak. Is it possible to anticipate seizure onset by non-linear analysis of intracerebral EEG in human partial epilepsies?. Rev Neurol (Paris) 155, pp. 454-6, 1999.

15. K. Lehnertz, R. G. Andrzejak, and J. Arnhold. Nonlinear EEG analysis in epilepsy: Its possible use for interical focus localization, seizure anticipation, and prevention. J Clin Neurophysiol 18, pp. 209-22, 2001

16. M. T. Rosenstein, J. J. Collins, and C.J. de Luca. A practical method for calculating the largest Lyapunov exponent from small data sets. Physica D 65, pp. 117-34, 1994.

17. H. Kantz. A robust method to estimate the maximal Lyapunov exponent of a time series. Phys Lett A 185, pp. 77-87, 1994.

18. R. Q. Quiroga, J. Arnhold, K. Lehnertz, and P. Grassberger. Kulback-Leibler and renormalized entropies: Applications to electroencephalograms of epilepsy patients. Phys Rev E Stat Phys Plasmas Fluids Relat Interdiscip Topics 62, pp. 8380-6, 2000.

19. R. G. Andrzejak, K. Lehnertz, F. Mormann, C. Rieke, P. David, and C. E. Elger. Indications of nonlinear deterministic and finite-dimensional structures in time series of brain electrical activity: Dependence on recording region and brain state. Phys Rev E 64, pp. 061907, 2001.

20. L. D. Iasemidis, P. Pardalos, J. C. Sackellares, and D. S. Shiau. Quadratic binary programming and dynamical system approach to determine the predictability of epileptic seizures. J Comb Optim 5, pp. 9-26, 2001.

21. R. G. Andrzejak, F. Mormann, G. Widman, T. Kreuz, C. E. Elger, and K. Lehnertz. Improved spatial characterization of the epileptic brain by focusing on nonlinearity. Epilepsy Res 69, pp. 30-44, 2006. 
22. M. Winterhalder, B. Schelter, T. Maiwald, A. Brandt, A. Schad, A. Schulze-Bonhage, et al. Spatio-temporal patient-individual assessment of synchronization changes for epileptic seizure prediction. Clin Neurophysiol 117, pp. 2399-413, 2006.

23. R. G. Andrzejak, F. Mormann, T. Kreuz, C. Rieke, A. Kraskov, and C. E. Elger. Testing the null hypothesis of the non-existence of the preseizure state. Phys Rev E 67, pp. 010901, 2003.

24. K. Lehnertz, and B. Litt. The first international collaborative workshop on seizure prediction: Summary and data description. Clin Neurophysiol 116, pp. 493-505, 2005.

25. M. A. Harrison, M. G. Frei, and I. Osorio. Accumulated energy revisited. Clin Neurophysiol 116, pp. 527-31, 2005.

26. R. Aschenbrenner-Scheibe, T. Maiwald, M. Winterhalder, H. U. Voss, J. Timmer, and A. Schulze-Bonhage. How well can epileptic seizures be predicted? An evaluation of a nonlinear method. Brain 126, pp. 2616-26, 2003.

27. T. Maiwald, M. Winterhalder, R. Aschenbrenner-Scheibe, H. U. Voss, A. Schulze-Bonhage, and J. Timmer. Comparison of three nonlinear seizure prediction methods by means of the seizure prediction characteristic. Physica D 194, pp. 357-68, 2004.

28. Y. C. Lai, M. A. Harrison, M. G. Frei, and I. Osorio. Inability of Lyapunov exponents to predict epileptic seizures. Phys Rev Lett 91, pp. 068102, 2003.

29. Y. C. Lai, M. A. Harrison, M. G. Frei, and I. Osorio. Controlled test for predictive power of Lyapunov exponents: Their inability to predict epileptic seizures. Chaos 14, pp. 630-42, 2004.

30. W. De Clercq, P. Lemmerling, S. Van Huffel, and W. Van Paesschen. Anticipation of epileptic seizures from standard EEG recordings. Lancet 361, pp. 971, 2003.

31. P. E. McSharry, L. A. Smith, and L. Tarassenko. Prediction of epileptic seizures: Are nonlinear methods relevant?. Nat Med 9, pp. 241-2, 2003.

32. M. Le Van Quyen, V. Navarro, J. Martinerie, M. Baulac, and F. J. Varela. Towards a neurodynamical understanding of ictogenesis. Epilepsia 44, pp. 30-43, 2003.

33. F. Mormann, T. Kreuz, C. Rieke, R. G. Andrzejak, A. Kraskov, and P. David. On the predictability of epileptic seizures. Clin Neurophysiol 116, pp. 569-87, 2005.

34. C. C. Jouny, P. J. Franaszczuk, and G. K. Bergey. Signal complexity and synchrony of epileptic seizures: Is there an identifiable preictal period?. Clin Neurophysiol 116, pp. 552-8, 2005.

35. R. Esteller, J. Echauz, M. D'Alessandro, G. Worrell, S. Cranstoun, and G. Vachtsevanos. Continuous energy variation during the seizure cycle: Towards an on-line accumulated energy. Clin Neurophysiol 116, pp. 517-26, 2005.

36. M. D’Alessandro, G. Vachtsevanos, R. Esteller, J. Echauz, S. Cranstoun, and G. Worrell. A multi-feature and multi-channel univariate selection process for seizure prediction. Clin Neurophysiol 116, pp. 506-16, 2005.

37. R. Q. Quiroga, A. Kraskov, T. Kreuz, and P. Grassberger. On the performance of different synchronization measures in real data: A case study on EEG signals. Phys Rev E 65, pp. 041903, 2002.

38. F. Mormann, R. G. Andrzejak, T. Kreuz, C. Rieke, P. David, and C. E. Elger. Automated detection of a pre-seizure state based on a decrease in synchronization in intracranial EEG recordings from epilepsy patients. Phys Rev E 67, pp. 021912, 2003.

39. F. Mormann, K. Lehnertz, P. David, and C.E. Elger. Mean phase coherence as a measure for phase synchronization and its application to the EEG of epilepsy patients. Physica D 144, pp. 358-69, 2000.

40. J. Arnhold, P. Grassberger, K. Lehnertz, and C.E. Elger. A robust method for detecting interdependences: Application to intracranially recorded EEG. Physica D 134, pp. 419-30, 1999.

41. H. P. Zaveri, M.G. Frei, S. Arthurs, and I. Osorio. Seizure prediction: The Fourth International Workshop. Epilepsy Behav 19, pp. 1-3, 2010.

42. A. L. Hodgkin, and A. F. Huxley. A quantitative description of membrane current and its application to conduction and excitation in nerve. Bull
Math Biol 52, pp. 25-71, 1952.

43. R. D. Traub. Neocortical pyramidal cells: A model with dendritic calcium conductance reproduces repetitive firing and epileptic behavior. Brain Res 173, pp. 243-57, 1979.

44. R. D. Traub. Simulation of intrinsic bursting in CA3 hippocampal neurons. Neuroscience 7, pp. 1233-42, 1982.

45. T. I. Netoff, R. Clewley, S. Arno, T. Keck, and J. A. White. Epilepsy in Small-World Networks. J Neurosci 24, pp. 8075-8083, 2004.

46. S. C. Ponten, F. Bartolomei, and C. J. Stam. Small-world networks and epilepsy: Graph theoretical analysis of intracerebrally recorded mesial temporal lobe seizures. Clin Neurophysiol 118, pp. 918-27, 2007.

47. J. J. Wright and D. T. Liley. Simulation of electrocortical waves. Biol Cybern 72, pp. 347-56, 1995.

48. R. L. Beurle. Poperties of a mass of cells capable of regenerating pulses. Trans Roy Soc (Lond) B 240, pp. 55-94, 1956.

49. H. R. Wilson, and J. D. Cowan. Excitatory and inhibitory interactions in localised populations of model neurons. Biophys J 12, pp. 1-24, 1972.

50. H. R. Wilson, and J. D. Cowan. A mathematical theory of the functional dynamics of cortical and thalamic nervous tissue. Kybernetik 13, pp. 55-80, 1973.

51. S. Amari. Homogeneous nets of neuron-likme elements. Biol. Cybern 17, pp. 211-20, 1975.

52. S. Amari. Dynamics of pattern formation in lateral-inhibition type neural fields. Biol Cybern 27, pp. 77-87, 1977.

53. W. J. Freeman. Mass Action in the Nervous System. New York NY: Academic Press; 1975.

54. B. Huberman, K. Kaneko, Ph. Maini, and Q. Ouyang. Comlex Physical, biophysical and econophysical systems. Proceedings of the 22nd Canberra International Physics Summer School. Singapore: World Scientific Publishing Co. Pte. Ltd; 2010.

55. F. H. Lopes da Silva, A. Hoeks, H. Smits, and L. H. Zetterberg. Model of brain rhythmic activity. Biol Cybern 15, pp. 27-37, 1974.

56. F. H. Lopes da Silva, and W. Storm van Leeuwen. The cortical alpha rhythm in dog: The depth and surface profile of phase. In: M. A. Brazier, H. Petsche, editors. Architectonics of the Cerebral Cortex. New York: Raven Press, pp. 319-33, 1978.

57. Lopes da Silva, W. Blanes, S. Kalitzin, J. Parra, P. Suffczynski, and D. N. Velis. Epilepsies as a dynamical disease of brain systems: Basic models of the transition between normal and epileptic activity. Epilepsia 44, pp. 72-83, 2003.

58. P. Suffczynski, S. Kalitzin, and F. H. Lopes da Silva. Dynamics of nonconvulsive epileptic phenomena modeled by a bistable neuronal network. Neuroscience 126, pp. 467-84, 2004.

59. B. H. Jansen, and V. G. Rit. Electroencephalogram and visual evoked potential generation in a mathematical model of coupled cortical columns. Biol Cybern 73, pp. 357-66, 1995.

60. V. D. Taranenko, and M. A. Rabtsevich. Neuronal responses of a chronically isolated slab of auditory cortex to intracortical stimulation during paroxysmal electrical activity in cats. Neurophysiology 16, pp. $1-8,1984$.

61. O. David, and K. J. Friston. A neural mass model for MEG / EEG: Coupling and neuronal dynamics. Neurolmage 20, pp. 1743-55, 2003.

62. P. L. Nunez. Electric fields of the brain: The neurophysics of EEG. 1st ed. New York: Oxford University Press; 1981.

63. D. T. Liley, P. J. Cadusch, and M. P. Dafilis. A spatially continuous mean field theory of electrocortical activity. Network 13, pp. 67-113, 2002.

64. F. Wendling, J. J. Bellanger, F. Bartolomei, and P. Chauvel. Relevance of nonlinear lumpedparameter models in the analysis of depth-EEG epileptic signals. Biol Cybern 83, pp. 367-78, 2000.

65. F. Wendling, F. Bartolomei, J. J. Bellanger, and P. Chauvel. Epileptic fast activity can be explained by a model of impaired GABAergic dendritic inhibition. Eur J Neurosci 15, pp. 1499-508, 2002.

66. D. L. Kimmel, and T. Moore. Temporal Patterning of Saccadic Eye 
Movement Signals. J Neurosci 27, pp. 7619-30, 2007.

67. D. T. Liley, and I. Bojak. Understanding the transition to seizure by modeling the epileptiform activity of general anesthetic agents. J Clin Neurophysiol 22, pp. 300-13, 2005.

68. P. Frogerais, J. J. Bellanger, and F. Wendling. Model-based measurement of epileptic tissue excitability. Conf Proc IEEE Eng Med Biol Soc 2007, pp. 1578-81, 2007.

69. F. Wendling, A. Hernandez, J. J. Bellanger, P. Chauvel, and F. Bartolomei. Interictal to ictal transition in human temporal lobe epilepsy: Insights from a computational model of intracerebral EEG. J Clin Neurophysiol 22, pp. 343-56, 2005.

70. E. Labyt, L. Uva, M. de Curtis, and F. Wendling. Realistic modeling of Entorhinal Cortex field potentials and interpretation of epileptic activity in the guinea-pig isolated brain preparation. J Neurophysiol 96, pp. 363-77, 2006.

71. D. Cosandier-Rimélé, J. M. Badier, P. Chauvel, and F. Wendling. A physiologically plausible spatiotemporal model for EEG signals recorded with intracerebral electrodes in human partial epilepsy. IEEE Trans Biomed Eng 54, pp. 380-8, 2007.

How to cite this article: Shayegh F, AmirFattahi R, Sadri S, Ansari-AsI K. A brief survey of computational models of normal and epileptic EEG signals: A guideline to model-based seizure prediction. J Med Sign Sens 2011;1:62-72

Source of Support: Nil, Conflict of Interest: None declared

\section{Author Help: Reference checking facility}

The manuscript system (www.journalonweb.com) allows the authors to check and verify the accuracy and style of references. The tool checks the references with PubMed as per a predefined style. Authors are encouraged to use this facility, before submitting articles to the journal.

- The style as well as bibliographic elements should be $100 \%$ accurate, to help get the references verified from the system. Even a single spelling error or addition of issue number/month of publication will lead to an error when verifying the reference.

- Example of a correct style

Sheahan P, O'leary G, Lee G, Fitzgibbon J. Cystic cervical metastases: Incidence and diagnosis using fine needle aspiration biopsy. Otolaryngol Head Neck Surg 2002;127:294-8.

- Only the references from journals indexed in PubMed will be checked.

- $\quad$ Enter each reference in new line, without a serial number.

- Add up to a maximum of 15 references at a time.

- If the reference is correct for its bibliographic elements and punctuations, it will be shown as CORRECT and a link to the correct article in PubMed will be given.

- If any of the bibliographic elements are missing, incorrect or extra (such as issue number), it will be shown as INCORRECT and link to possible articles in PubMed will be given. 